\title{
Management of Tinnitus In Covid-19 Outbreak- A Comparative Study Between Mindfulness Based Tinnitus Stress Reduction And Tinnitus Retraining Therapy
}

\author{
Nikita Chatterjee ${ }^{1^{*}}$ \\ Durba Chattopadhyay² \\ Indranil Chatterjee ${ }^{3}$
}

\begin{abstract}
Background: Covid-19 pandemic has caused a profound impact on the lives of people and has given rise to many mental health issues like anxiety and stress which eventually has given rise to bothersome tinnitus. In case of people having pre-existing tinnitus, the pandemic has raised the problem of tinnitus and the co-morbid issues related to tinnitus and severely affected the overall quality of life. Hence, we need to find a mean for home-based management of tinnitus and its associated problems.

Objective: To understand the mechanism of mental health and to compare between the mindfulness-based tinnitus reduction and tinnitus retraining therapy on android-based application.

Design: Comparative and Experimental design.

Method: The study was conducted on 60 participants and was divided into two groups. Detailed audiological assessment, tinnitus assessment and Tinnitus Handicap Inventory (THI) and Tinnitus Cognitions Questionnaire (TCQ) were administered. Group-I was provided with tinnitus retraining therapy and group-II was provided with mindfulness based tinnitus stress reduction. After the post therapy evaluation the data were compiled for statistical analysis in SPSS software 20.0. Results: It was found that there were significant difference between the pre and post therapy scores of TRT and MBTSR in both THI and TCQ $(p<0.001)$.

Conclusion: Home-based management of tinnitus during the COVID-19 pandemic using both TRT and MBTSR were highly successful and had similar results MBTSR was found to be more useful as it had better outcome in reducing the tinnitus related annoyance and shown better improvement in QOL examination.
\end{abstract}

Keywords: COVID-19 Pandemic, Tinnitus-Retraining Therapy, Mindfulness-based tinnitus stress reduction.

${ }^{1}$ Department of audiology and speech language pathology, AYJNISHD,R.C., Kolkata

${ }^{2}$ Department of Audiologist and speech language pathologist, Indian Railways, India

${ }^{3}$ Lecturer in speech and hearing, department of Audiology (Head of the department), AYJNISHD,R.C., Kolkata 


\section{INTRODUCTION}

The newly identified novel coronavirus, belonging to the same family of "Severe acute respiratory syndrome" (SARS) and "Middle East respiratory syndrome" (MERS) coronavirus ; Sars-CoV-2, was first reported in Wuhan, China, in late 2019'. Covid-19 pandemic has a rapid and profound impact on every part of our life, from the way people work, live, shop, socialize, and plan for the future. In current scenario, the total number of confirmed cases has hiked from December, 2019 to September 2020, to more than 25.1 million where India has the largest number of confirmed cases in Asia and has the second highest number of cases in world after United States ${ }^{2}$. The necessary public health care actions taken to control the spread of Covid-19 such as, social distancing, quarantine and lockdown can also make people feel isolated and lonely and can increase stress and anxiety ${ }^{3}$. In these circumstances, when the level of stress in general population has increased to a great extent, having a serious condition like Tinnitus, which has a strong correlation with stress ${ }^{4}$, increases the problem even further. Tinnitus is defined as an abnormal acoustical sensation perceived by an individual in the absence of any external sound source. Tinnitus is one of the most common conditions experienced by people; however, interestingly, this condition is non-bothersome to most people. Furthermore, in case of bothersome tinnitus, individualistic the type and extent of the difficulties varies from person to person. Persistently intolerable or sufficiently bothersome tinnitus can cause functional impairment in cognitive processing5, mood 5, hearing, sleep, and concentration ${ }^{5}$; giving rise to frustration, depression and anxiety ${ }^{6}$, severely affecting the on quality of life ${ }^{7}$. In India, approximately 4.5 million people are affected with tinnitus, unfortunately the exact data of etiology and prevalence is not available ${ }^{8}$. However, prevalence of tinnitus correlates with the severity and frequency characteristics of hearing loss $^{9}$. Tinnitus has been found to impact many different domains of functioning catering to a need of a multidisciplinary approach for its management in order to adequately help the patients. But at this point, no universal "cure" for tinnitus and its physical or emotional consequences has been identified ${ }^{10}$. In search of an efficient technique to treat tinnitus, researchers have taken into account the ancient method of therapy to treat tinnitus and its concomitant physical and emotional problems. Because individuals with tinnitus share many similarities with those suffering pain, an efficacious treatment strategy for pain management should be implemented in tinnitus management protocol ${ }^{10}$. In this current era, when it is difficult for the patients to reach hospital and institutes for tinnitus management and therapy, the digital platform has been implemented for home-based management of tinnitus by using the app based relaxation and retraining. Tinnitus sound therapy is one of the most useful method for reliving and managing tinnitus. As a part of a tinnitus management program, there are apps which offer a combination of sound therapy for masking the tinnitus, relaxing exercises, meditation, and guidance.

Mindfulness-based meditation is another such home -based management protocol that can be implemented with its power to bring relief to troubling symptoms is a therapy that shows great promise in the present-day treatment of disease and management of health. This technique has been conceptualized by Jon KabatZinn ${ }^{11}$, who developed an 8-week treatment program called Mindfulness Based Stress Reduction (MBSR). A mindfulness practices imply trains the mind to notice the human tendency to allow the mind to wander and to gently, without judgment, guide the mind back to the only moment that is; the present ${ }^{11}$. Recent research has shown that mindfulness-based approaches to living with tinnitus are effective in helping people modify their perception, leading to reduced tinnitus bother, less emotional struggle, and a greater sense of well- being. Specifically, Mindfulness Based Tinnitus Stress Reduction (MBTSR), an 8-week skill- building program developed and researched at University of California San Francisco (UCSF), teaches participants how to develop more healthful and positive ways of relating to the unpleasant sensation. MBTSR includes in- depth tinnitus education and mindfulness skill building. Each week's class integrates elements of deep breathing, gentle yoga, relaxation, and meditation to help people develop new, more effective ways to relate to the experience of tinnitus and stress in their daily lives 10 . The aim is not to change or judge the body, which may be difficult for people experiencing tinnitus. Patients are often told "don't think about it" or "just distract yourself." But trying not to think about one's tinnitus usually doesn't work. In fact, avoiding and pushing away these thoughts can make the perception of tinnitus even good.

Need of the Study: In this new normal era, mask and digital platform has become a part of our life. This Covid-19 Pandemic has caused a great deal of damage to the mankind in various arenas like social, emotional, physical, economic, etc which has hiked the level of stress and given rise to the emergence of mental illness along with triggering other health problems like tinnitus, that were already present in the pre-pandemic time. But, in this current scenario, it is difficult for the patients to visit the clinics and institutes for management of tinnitus due to the state-wide and nation-wide lockdown imposed for control the transmission and spread of Sars-CoV-2. Hence, in this situation, it is highly required to develop a home-based protocol, which has equal proficiency as the audiological clinics, for the management of tinnitus as well as the co-morbid and triggering factors that has emerged in the time of pandemic and has raised the problem of tinnitus even more.

Aim: This study aims to understand the mechanism of mental health and to compare between the mindfulnessbased tinnitus reduction and tinnitus retraining therapy on android-based application.

Methods: The study was done on 60 subjects with normal hearing sensitivity (SDS within the range of $80 \%$ to 100\%) 
and the age range between 20 and 60 years (mean age $=$ 45.02years, $S D=3.76$ ); suffering from chronic tinnitus. All participants had android phones having minimal technical knowledge. These participants were divided into two groups. Group 1 consisted of 30 subjects with 15 males (mean age $=43.64$ years, $S D=3.34$ ) and 15 females (mean age $=46.43$ years, $S D=3.97$ ), who were provided through mindfulness-based tinnitus stress reduction (MBTSR) using MBSR App. and Group 2 consisted of 30 subjects that includes 15 males (mean age $=44.34$ years, $\mathrm{SD}=4.34$ ) and 15 females (mean age $=45.67$ years, $\mathrm{SD}=3.38$ ), whom TRT was provided through the app.

Tools or instruments: Diagnostic pure-tone audiometer (Maico MA 53 calibrated) with TDH 39 headphones, GSI 39 Middle Ear Analyzer were used for the study. Personalized Android based Tinnitus Apps Resound Relief, TCQ and THI for obtaining the pre-therapy status of percieved tinnitus and its effect on the participants.

\section{Procedure:}

Phase 1: Written consent was taken for all of the participants participated in this study. Ethical clearance was obtained from the institutes committee.

Phase 2: Next following audiometric assessment, detailed tinnitus assessment was carried out by using ASHA guidelines, 2005, with reference to Pitch matching (65\% had unilateral tonal tinnitus, $30 \%$ had noisy tinnitus and $5 \%$ had multicomponent tinnitus-unable to match), loudness matching (mean- $45.78 \mathrm{dBHL}, \mathrm{SD}-6.87 \mathrm{~dB}$ ), minimum.

Phase 3: For group 1, mindfullness-based tinnitus stress reduction (MBTSR) therapy was carried out by using MBSR protocol of stress reduction and the participants were taught to develop more healthful and positive ways of relating to the unpleasant sensation of tinnitus. Online counselling and MBTSR, including in- depth tinnitus education and mindfulness skill building were carried out for total 8 sessions in the period of 2 months by using video calling facility. Each week's class integrates elements of deep breathing, relaxation, and meditation (formal and informal) to help people develop new, more effective ways to relate to the experience of tinnitus and stress in their daily lives10. Participants were taught to allow unpleasant thoughts, feelings, or sensations about tinnitus to simply go in and out of their awareness without having to do battle with them by .The formal meditation portion of the program involves practice in sitting meditation, body scan, and gentle mindful yoga, whereas the informal meditation integrates these practices into everyday life as a coping tool for dealing with intense physical symptoms (such as tinnitus) and difficult emotions associated with tinnitus 10. Clinicians were trained to transfer the mindfulness based tinnitus reduction skills to the participants and each session were lasted for 2hours. Participants were not counseled to "not to think about tinnitus" rather told to adapt the thoughts, sensation and feeling hence, allowing the body to unlock its ability habituate. Participants were asked to carry out the activities regularly at home for a total of 60 days, which were taken equivalent 60 sessions; and give their report on the planned session via video-call before the beginning of each video-call MBTSR session.

Phase 4: The subjects of group 2 had installed ReSound Relief Tinnitus App and they were shown and instructed for its usage. They reported once in a week to give us the data of their usage on online panel and they were counseled for a total of 8 sessions in the period of two months. They have used apps in total of 60 days, which was taken as 60 sessions equivalent for the study. TRT was used in three significant therapeutic steps such as Tinnitus intake interview, use of android based tinnitus apps (resound relief) by using headphones of personalized smartphones and directive counselling. Sound therapy; for reducing the attention drawn to the tinnitus loudness of tinnitus was combined with directive counselling; for improving the patients well-being, day-to-day life, social interactions, and working abilities of the participants 12 . All the participants were trained to set the mixing point at which they were able to here both the perceived tinnitus tone and the masking tone from the app while the levels were set on the Resound Relief tinnitus app.

Phase 5: All the participants were assessed using THI and TCQ to obtain the post therapy measures on self-perceived tinnitus handicap and cognition. Also, participants were retested after 1-month follow-up by using TCQ and THI to check the transfer and maintenance.

Phase 6: All data were statistically analyzed by using SPSS version 20.0 by means of measures of central tendency (Mean, Standard Deviation) and Paired t test.

\section{RESULTS}

The purpose of the study was to find out the alternative mean for management of tinnitus and tinnitus related stress in the Covid-19 pandemic era using the mindfulness-based tinnitus reduction and tinnitus retraining therapy at home using Android based digital platform and to compare between the mindfulness-based tinnitus reduction and tinnitus retraining therapy. The comparison between the pre and post therapy data of MBTSR and TRT in Bangla-THI and TCQ was obtained and analyzed in SPSS software version 20.0.

\section{Comparison between pre and post TRT and MBTSR therapy in Bangla-THI}

The comparison between pre-therapy and post-therapy data of TRT and MBTSR in Bangla-THI revealed that there was extremely significant statistical difference between the pre-therapy and post therapy status of the participants $(p<0.0001)$ which suggests that their were significant reduction in the scores of the participants from catastrophic handicap to moderately handicapped in functional, emotional and catastrophic subscales of $\mathrm{THI}$ and the overall improvement in tinnitus related quality of life of the participants with chronic tinnitus after the 8 
Table 1: Comparison between the mean THI scores of pre-therapy and post-therapy TRT and MBTSR.

$\begin{array}{ccc}\text { THI score } & \text { Pre-therapy } & \text { Post-therapy } \\ \text { TRT } & 98 & 43.64 \\ \text { MBTSR } & 97 & 37.9\end{array}$

Table 2: Comparision between the mean TCQ scores of pre-therapy and post-therapy TRT and MBTSR.

$\begin{array}{ccc}\text { TCQ score } & \text { Pre-therapy } & \text { Post-therapy } \\ \text { TRT } & 92 & 33 \\ \text { MBTSR } & 93 & 32\end{array}$

sessions of TRT and MBTSR therapy which can be seen in the following Table 1.

\section{Comparison between pre and post TRT and MBTSR therapy in TCQ}

Significant statistical difference was obtained between the pre-therapy and post-therapy scores of TRT and MBTSR $(p<0.0001)$ in TCQ as found in table-2. This suggests that the positive thoughts related to tinnitus was significantly less and negative thoughts related to tinnitus was significantly higher with overall higher scores on the whole questionnaire before the TRT and MBTSR therapy. After 8 sessions of therapy, the participants perceived tinnitus as the "small problem" with significantly increased scores on positive subscales and lower scores on negative sub-scales and significant reduction in the overall scores of the whole questionnaire. Hence, the tinnitus related stress was found to be significantly reduced with increased level of positive thoughts and coping mechanism and overall tinnitus-related quality of life Table 2

\section{RESULTS AND DISCUSSION}

This study aimed at understanding the mechanism of mental health and to find out a home-based regimen for the treatment of tinnitus and tinnitus-related mental health issues like stress, anxiety and tension. Hence, this comparative study was done to find out the effectiveness and of mindfulness-based tinnitus stress reduction and tinnitus retraining therapy on android-based application and to find out any possible differences between them. The findings of table-1 suggested that after 2months of home-based management of tinnitus by using TRT for Group-I participants and MBTSR for group-II participants, there was significant decrements in the severity scores of THI from catastrophic handicap to moderate handicap. Also, the THI scores of group-I participants who were given TRT had resemblance with THI scores of groupII participants who were given MBTSR therapy. This finding suggested that the 8-weeks (2months) course of both TRT and MBTSR had significant and similar impact on functional, emotional and catastrophic aspects of participants living with chronic tinnitus. In consonance to this finding, a pilot study showed that 8-week course of MBTSR can be effectively used for the intervention of tinnitus by decreasing the tinnitus annoyance and its impact on daily living of their participants ${ }^{12}$ to see the sustained benefits of mindfulness-based tinnitus stress reduction therapy on patients with chronic tinnitus suggested that the benefits from the MBTSR therapy or training can be sustained for at least 12 months in adults with chronic tinnitus and decline in THI score can be observed after long-term usage of MBTSR in patients with chronic tinnitus ${ }^{13}$.

In Table-2, it can be seen that there was significant decline in the TCQ scores after 8 weeks of TRT and MBTSR training. It was observed that Group-II participants who underwent MBTSR for 8 weeks has more positive thoughts and better control over the unpleasant and bothersome thoughts related to tinnitus. This can be resulted from use of formal and informal meditation as an integral part of MBTSR training. Hasenkamp and Barsalou (2012), reported in their study, that participants with more experience of meditation exhibit increased connectivity in cortical networks (attention) as well as between attentional regions and default mode network ${ }^{14}$. In another study, examining the effectiveness of MBSR therapy on subjective bother and neural connectivity in chronic tinnitus suggested that MBSR program can result in neuroplastic changes in functional connectivity in networks responsible for attention, decision-making, and somatosensory input ${ }^{15}$.

\section{CONCLUSION}

In the present study, it was found that, the Covid-19 pandemic have caused ineluctable changes in terms of physical and mental wellbeing of person with or without any underlying mental health issues. And it has caused significant increment in the perception of tinnitus and tinnitus related stress, anxiety and negative thoughts leading to depression. Also, it was found that, in this pandemic situation, when the institution based management of tinnitus is difficult, use of digital panel for the treatment of tinnitus focusing on the TRT and MBTSR which has shown positive outcome.

\section{CONFLICT OF INTEREST}

There are no conflicts of interest in terms of the present study.

\section{REFERENCES}

1. The Novel Coronavirus Pneumonia Emergency Response Epidemiology Team. The epidemiological characteristics of an outbreak of 2019 novel coronavirus diseases (COVID-19): China. 2020;2:113-22. 
2. World Health Organization (WHO) Eastern Mediterranean Regional Office. Laboratory confirmed cases of MERS reported in Eastern Mediterranean Region. 2020.

3. Banerjee D. How COVID-19 is overwhelming our mental health. Nat India. 2020.

4. Mazurek B, Haupt H, Olze H, Szczepek JA. Stress and tinnitus- from bedside to bench and back. Front syst neurosci. 2012;6;47.

5. Moring J, Bowen A, Thomas J, Bira L. The emotional and functional impact of the type of tinnitus sensation. $J$ of clinical psychology. 2016;23:310-18.

6. Durai M, Searchfield G. Anxiety and depression, personality traits relevant to tinnitus: a scoping review. Interntional journal of audiology. 2016;55:605-15.

7. Kennedy V, Wilson C, Dafydd S. Quality of life and tinnitus. Audiological medicine. 2004;2:29-40.

8. Vempati A, Prakash SGR, Rathna SB, Sandhya K. Management options for individuals with tinnitus - a review. Int J Sci Res. 2013;2:290-4.
9. Sindhusake D, Golding M, Newall P, Rubin G, Jakobsen $K$, Mitchell $P$. Risk factors for tinnitus in a population of older adults: the blue mountains hearing study. Ear Hear. 2003;24:501-7.

10. Gans JJ. Mindfulness-based tinnitus therapy is an approach with ancient roots. The hearing journal. 2010;63:52-6.

11. Kabat-Zinn J, Massion AO, Kristeller J. Effectiveness of a meditation-based stress reduction program in the treatment of anxiety disorders. Am J Psych. 1992;149:936-43

12. Tyler RS. Neurophysiological models and treatments for tinnitus. Tinnitus treatment: clinical protocols. 2006;1-22.

13. Gans JJ, Cole MA, Greenberg B. Sustained benefits of mindfulness-based tinnitus stress reduction in adults with chronic tinnitus: a pilot study. Mindfulness. 2015;6:1-4.

14. Hasenkamp W, Barsalou LW. Effects of meditation experience on functional connectivity of distributed brain networks. Front hum neurosci. 2012;6:3-8.

15. Roland LT, Lenze EJ, HArdin FM. Effects of mindfulness based stress reduction therapy on subjective bother and neural connectivity in chronic tinnitus. Otolaryngol head neck surg. 2015;152:919-26. 